\title{
NUMERICAL ANALYSIS OF HEAT TRANSFER OVER HORIZONTAL ROTATING CYLINDER IN PRESENCE OF AXIAL FLOW
}

\author{
Shabresh A \\ Department of Aerospace Engineering \\ Alliance University, Bangalore, Karnataka, India
}

\author{
Keerthana N \\ Department of Aerospace Engineering \\ Alliance University, Bangalore, Karnataka, India
}

\author{
Manjunath SV \\ Department of Aerospace Engineering \\ Alliance University, Bangalore, Karnataka, India
}

\begin{abstract}
In the recent years, enhanced boiling heat transfer has emerged as a potential research area to meet the challenges of technological developments in cooling of electronic components, nuclear fuel rods, nuclear reactors etc. In this paper, we numerically analyze the influence of rotation on horizontal cylindrical heating element made of copper in the medium of water around atmospheric conditions. The critical Reynolds number is influenced by Prandtl number and Nusselt number based on the cylinder diameter for stationary boiling. Boiling regimes has been divided into sub-critical and super critical regions based on critical Reynolds number. Effect of rotation on heat transfer coefficient (HTC) in these regions are investigated. Numerical analysis has been carried out using MATLAB for solving boiling equation of Lienhard which is correlated for rotational effects in different regimes by considering Weber number and Froude number.
\end{abstract}

Keywords - Rotating cylinder, Nucleate boiling, conjugate heat transfer

\section{INTRODUCTION}

A cylindrical rod made up of copper with length $=0.2 \mathrm{~m}$, and diameter of $0.02 \mathrm{~m}$ is taken and is placed inside the water medium and can rotate in various speed. The rotational effects of the convective heat transfer of the cylinder has been investigated. The copper cylinder is rotated in the rotating Reynolds number of $0-2.6 \times 10^{5}$ and the heat flux densities of $2.5 \times 10^{4} \mathrm{w} / \mathrm{m}^{2}$ to $8.2 \times 10^{4} \mathrm{w} / \mathrm{m}^{2}[1]$.

$$
R e_{c}=\frac{N u_{s b}}{0.12 \times \operatorname{Pr}^{\frac{1}{3}}}
$$

The rotation has no effects on the heat transfer coefficients in low-moderate speeds [2].
In this speed for,

$$
0 \leq\left(\operatorname{Re}=\frac{D^{2} \omega}{2 \gamma}\right)<0.35\left[\operatorname{Re} c=\left(\frac{N u_{s, b}}{0.12(\mathrm{PP}))^{1 / 3}}\right)^{3 / 2}\right]
$$

where $N u_{s b}$ is the Nusselt number based on the cylinder diameter for stationary boiling. The existing correlations are been used to find the heat transfer rate. At very high rotary speeds boiling ceased and forced convection became the only mode of heat transfer [3]. In this region, for $\operatorname{Re} \geq 1.35 R e_{c}$, the experimental data are correlated well by

$$
\mathrm{Nu}=0.12\left(\frac{D^{2} \omega}{2 \gamma}\right)^{2 / 3}(\operatorname{Pr})^{1 / 3}
$$

The region below the critical Reynolds number is termed as sub-critical region. The region after the critical Reynolds number is termed as super-critical region. In sub-critical region the HTC will decrease with increase in the Reynolds number and approaches a minimum value and tends to zero. At critical Reynolds number the bubble formation discontinues, and the boiling heat transfer due to the rotation will gets ceased [4]. In super-critical region with increase in Reynolds number the HTC will further go down, and heat flux in super-critical region is very least.

For a cylinder submerged in water the forced convection heat transfer can be induced either by increasing the linear velocity of the water or by rotating the cylinder in specific Reynolds number [5]. The heat transfer due to the rotation of the cylinder depends upon the angular velocity in which the cylinder is rotating. The heat transfer coefficient (HTC) will tends to decrease as the frequency of bubble formation decreases [6]. Hence the HTC will decrease with increase of rotation speed and finally tends to zero. The boiling heat transfer which occurs due to the formation of bubbles from the nucleate site when the surface temperature is higher than that of the saturation temperature, will gets ceased and the bubble 


\section{International Journal of Engineering Applied Sciences and Technology, 2020 \\ Vol. 4, Issue 12, ISSN No. 2455-2143, Pages 301-304 \\ Published Online April 2020 in IJEAST (http://www.ijeast.com)}

formation halts. Then the heat transfer occurs only due to the forced convection of the water.

\section{LITERATURE SURVEY}

The heat transfer at the outer surface of a rotating cylinder, both in a still environment or in axial flow, has been studied by various authors for different flow regimes. The correlation in boiling heat transfer from a rotating surface was given by Nicol and McLean[7]. They correlated their results in terms of boiling mechanism, which differed above and below the critical speed. The critical speed was found to be $150 \mathrm{rpm}$ for a 0.922 inch diameter copper cylinder boiling in water. Each run was performed at a constant rotary speed while the power input to the test section was varied at increments of approximately 200 watts. The surface finish of the cylinder was not specified. They found that an increase in the rate of heat transfer per unit temperature difference occurred for rotational speeds up to $150 \mathrm{rpm}$. For rotational speeds greater than this value, the rate of heat transfer decreased.

Anderson and Saunders [8] measured the heat transfer from horizontal cylinders, 1.0, 1.8 and 3.9 inches in diameter, each 2 feet long, rotating in still air. The material of the cylinders was not mentioned. They showed that the Nusselt number is almost independent of the Reynolds number for rotational speeds from zero to a critical value. The critical Reynolds number was found to be equal to

$$
\operatorname{Re}_{c r}=1.09(\mathrm{Gr})^{1 / 2}
$$

Above the critical Reynolds number, it was found that the Grashof number had a negligible effect on the rate of heat transfer. They correlated their results in this region by replacing the buoyancy acceleration term $(\beta g \Delta T)$ in the free convection equation for a horizontal surface,

$$
\mathrm{Nu}=0.14(\mathrm{Gr} P r)^{1 / 3}
$$

using cylinder diameter as the characteristic length, with a corresponding centrifugal term $\left(2 U^{2} / \mathrm{D}\right)$ thus yielding

$$
\mathrm{Nu}=0.10(\mathrm{Re})^{2 / 3}
$$

Equation mentioned above was later rearranged into the following form by Becker by introducing the Prandtl number in order to be applied to the general case of any liquid [9],

$$
\mathrm{Nu}=0.111 e^{2 / 3}\left(\mathrm{Prin}^{1 / 3}\right.
$$

Etemad[10] investigated the heat transfer and flow around horizontal copper and bakelite cylinders, 2 and 2.5 inches in diameter, each $85 / 8$ inches long, rotating in air between a range of Reynolds numbers from 0 to 65400 . He found that for Reynolds numbers below 1000 the Nusselt numbers depend almost entirely on the Grashof numbers.

Dropkin and Carmi [11] studied experimentally the convective heat transfer from two horizontal nickel-plated copper cylinders, 4.5 and 2.5 inches in diameter and each 21 inches long, rotating in air for Reynolds numbers up to 433000 . For Reynolds numbers larger than 15000 they suggested the following heat transfer correlation,

$$
\mathrm{Nu}=0.073(\mathrm{Re})^{0.7}
$$

\section{GOVERNING EQUATIONS}

The correlation for heat transfer of rotating surface below critical speed, the effects of rotation were correlated by the inclusion of a Weber-Reynolds number term in the boiling equation of Lienhard which took the form [12],

$$
\mathrm{Q} / \mathrm{A}=235(\Delta T)^{1.76}(k \cdot l)(\operatorname{Pr})^{1 / 3}\left(1+178(\mathrm{We} / \mathrm{Re})^{0.63}\right)
$$

Above the critical speed the same basic equation was modified to include a Froude number which gave the correlating equation[13],

$$
Q / A=520(\Delta T)^{1.76}(k . l)(P r)^{1 / 3}(F r)^{-0.186}
$$

The above equations are numerically simulated to understand the behavior of heat transfer from the cylinder under a range of Reynolds number.

\section{METHODOLOGY}

The boiling heat transfer analysis over a cylinder placed horizontally is studied. Initially, the material of the heating element should be selected to study the effect of rotation on the heat transfer co-efficient (HTC). Material property will impart change in HTC, hence suitable material for the study should be taken. The medium in which we place the heating element will also impart changes in HTC, hence suitable medium should be selected based on the requirement of rate of heat transfer [14]. The rotation of cylinders will be taken in terms of RPM which will be changed corresponding to the angular speed ' $\omega$ '. We had considered a horizontal cylindrical heating element made of copper placed in water medium under standard atmospheric conditions. It has length of $0.2 \mathrm{~m}$ and diameter of $0.02 \mathrm{~m}$ (D). MATLAB is used to solve boiling equation of Lienhard which is correlated for rotational effects in different regimes by considering Weber number and Froude number.

\section{RESULTS \& DISCUSSION}

We had analysed the heat transfer over horizontal cylinder in three cases using MATLAB as solver.

CASE 1: When the cylinder of diameter ' $D$ ' is rotating at ' $\omega$ ' angular speed and zero linear velocity of water ' $\mathrm{V}$ '

CASE 2: When cylinder having diameters of D, 2D, 4D, 6D, $8 \mathrm{D}, 10 \mathrm{D}$, are rotating at ' $\omega$ ' angular speed and zero linear velocity of water ' $v$ '

CASE 3: When cylinder of diameter D, 2D, 4D, 6D, 8D, 10D, are having zero angular speed ' $\omega$ ' and finite linear velocity ' $v$ ' 


\section{International Journal of Engineering Applied Sciences and Technology, 2020 \\ Vol. 4, Issue 12, ISSN No. 2455-2143, Pages 301-304 \\ Published Online April 2020 in IJEAST (http://www.ijeast.com)}

\section{CASE 1}

Fig A. Variation of heat flux vs RPM of rotating cylinder having diameter $\mathrm{D}(0.02 \mathrm{~m})$, at zero velocity of water

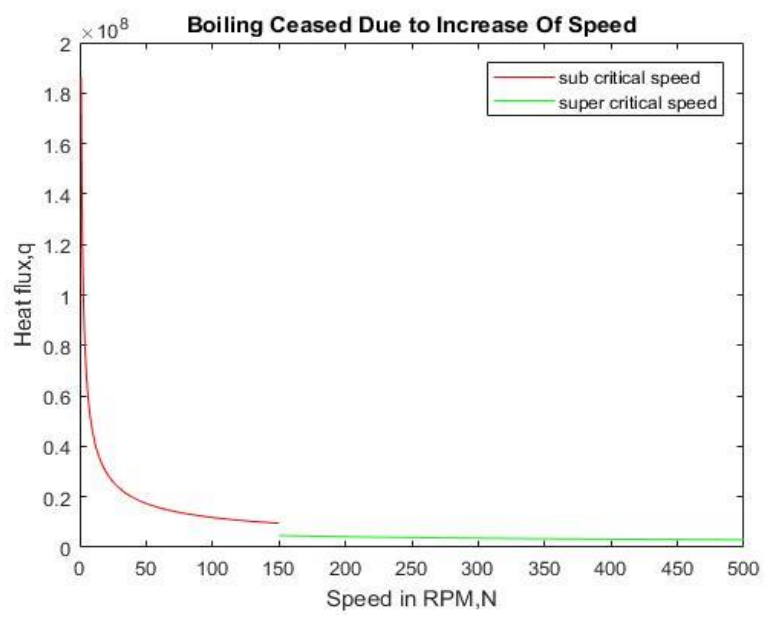

Fig B. Variation of heat flux with Reynolds number in subcritical region for rotating cylinder of diameter D

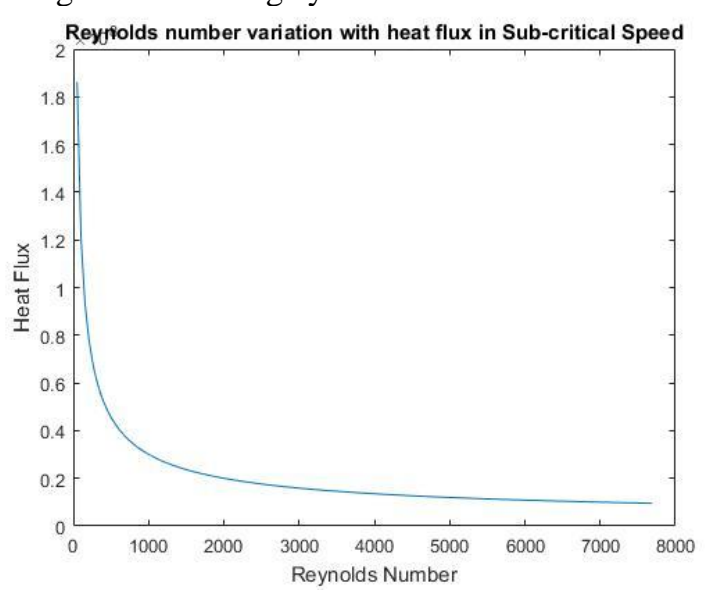

CASE 2

Fig C. Variation of HTC with RPM of cylinder having diameter D, 2D, 4D, 6D, 8D, 10D

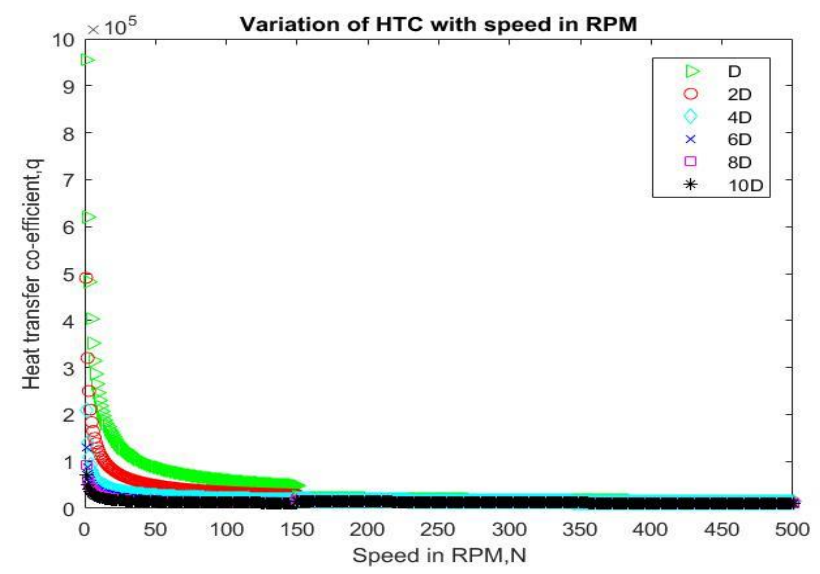

Fig D. Variation of HTC with RPM of cylinder of diameters $\mathrm{D}, 2 \mathrm{D}, 4 \mathrm{D}, 6 \mathrm{D}, 8 \mathrm{D}, 10 \mathrm{D}$ in sub-critical region $(\mathrm{RPM}<150)$

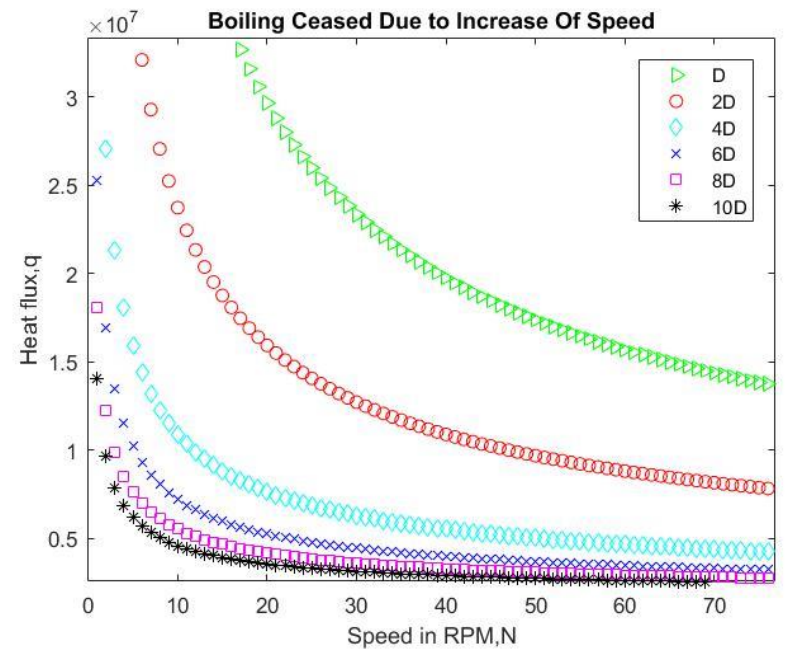

Fig E. Variation of HTC with RPM of cylinder of diameters $\mathrm{D}, 2 \mathrm{D}, 4 \mathrm{D}, 6 \mathrm{D}, 8 \mathrm{D}, 10 \mathrm{D}$ in super-critical region (RPM > 150)

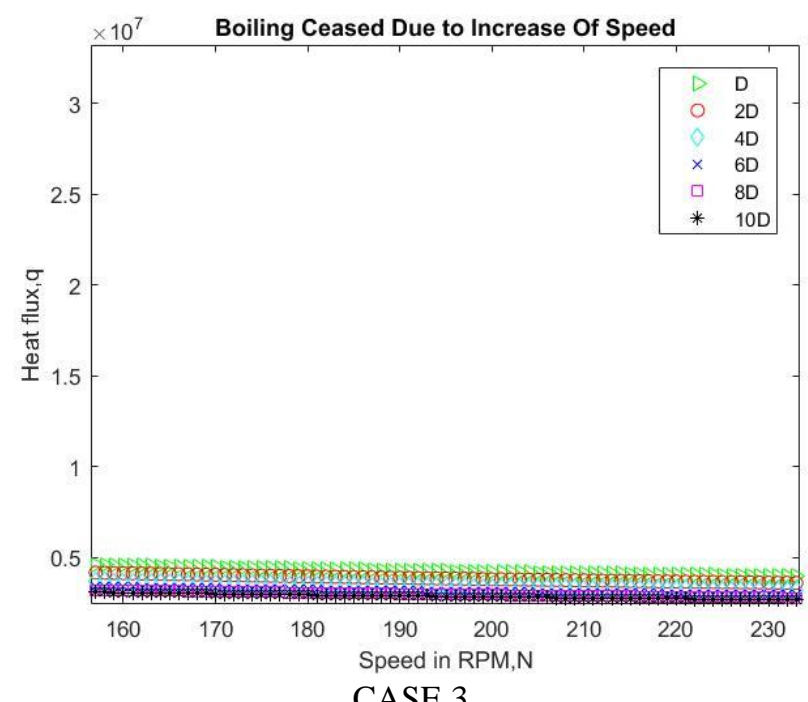

Fig F. Variation of HTC in forced convection of water with increasing velocity for cylinder of diameters $\mathrm{D}, 2 \mathrm{D}, 4 \mathrm{D}, 6 \mathrm{D}$, $8 \mathrm{D}, 10 \mathrm{D}$

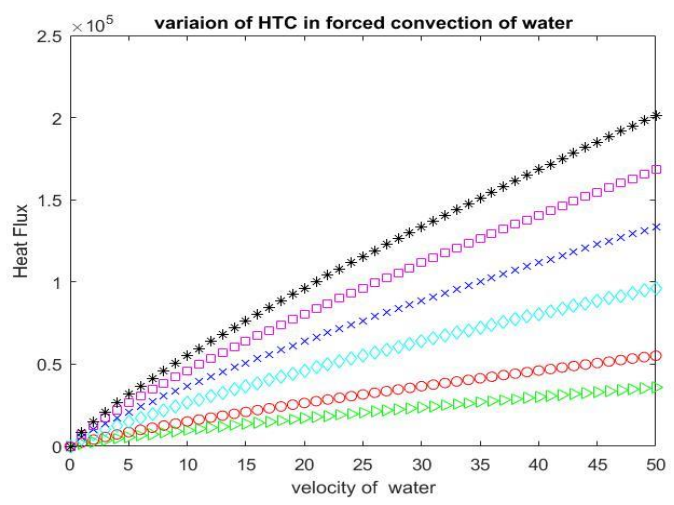




\section{International Journal of Engineering Applied Sciences and Technology, 2020 \\ Vol. 4, Issue 12, ISSN No. 2455-2143, Pages 301-304 \\ Published Online April 2020 in IJEAST (http://www.ijeast.com)}

Figure A, B represents the variation of heat flux with the RPM of horizontal rotating cylinder. Heat flux reduces with increase in RPM upto a critical point, which is determined as subcritical region. Sub critical region converts to super critical region, at a critical point of 150 RPM for cylinder of $0.02 \mathrm{~m}$ diameter and $0.2 \mathrm{~m}$ length having copper as outer material. In super critical region, the heat flux reduces and is nullified. Bubble growth from nucleate sites reduces, hence boiling ceases. It infers, there is no significant use of rotation of cylinder in enhancing heat transfer. Figure C, D, E, increase of diameter of cylinder reduces heat flux with increase of RPM, thus cylinder having minimum possible diameter gives more heat flux. Figure F represents the variation of heat flux with linear velocity of water. As the boiling ceases, the heat transfer occurs only due to forced convection of water.

\section{CONCLUSION}

This paper concerns about the influence of rotation on heat transfer from horizontal cylindrical heating element made of copper in water medium. Numerical analysis of heat transfer in sub critical region and super critical region, enables us to understand the nature of heat transfer process [15]. We had discussed three cases under these regimes,

1. In first case, the cylinder is rotating at ' $\omega$ ' angular velocity and zero linear velocity of water ' $v$ '; here HTC tends to decrease as Reynolds number increases upto a certain point where critical Reynolds number comes into picture. Such that, bubble growth rate decreases as Reynolds number becomes more than critical Reynolds number. Hence, boiling ceases and no heat transfer occurs in higher Reynolds number due to rotation.

2. In second case, the cylinder having diameters of D, 2D, 4D, $6 \mathrm{D}, 8 \mathrm{D}, 10 \mathrm{D}$, are rotating at ' $\omega$ ' angular speed and zero linear velocity of water ' $v$ '; here HTC tends to decrease for increasing diameter of cylinder with increase in RPM. Thus, it shows that cylinder with minimum diameter enhances boiling heat transfer under rotation.

3. In third case, the cylinder of diameter D, 2D, 4D, 6D, 8D, $10 \mathrm{D}$, are having zero angular speed ' $\omega$ ' and finite linear velocity ' $v$ '; here HTC tends to increase for increasing diameter of cylinder with increase in linear velocity of water. Thus, it shows that cylinder having larger diameter enhances boiling heat transfer under axial flow of water.

Research and development in heat transfer phenomena will impart major role in increasing rate of cooling efficiencies in nuclear reactor and fuel rods[16]. Effects of surface texture, gravity, surface roughness, positions of cylinder will also influence heat transfer rate and should be studied further.

\section{REFERENCES}

[1]. G.B. Wallis et al.(1982), Review, Theoretical Models of Gas-liquid Flows, Journal of Fluids Engineering, Vol. 104, (pp 279-283).
[2]. Moshkin NP and Sompong et al.(2010), Numerical simulation of heat transfer and fluid flow over two rotating circular cylinders at low Reynolds number, Heat TransferAsian Res, Vol 39(pp 246-261).

[3]. G. Ribatski et al.(2002), Theoretical and Experimental Analysis of Pool Boiling of Halocarbon Refrigerants, Doctoral thesis.

[4]. Shih-I Tang et al.(1970), A study of nucleate boiling heat transfer over a horizontal rotating cylinder, University of Windsor, Electronic theses and dissertations.

[5]. Manjunath S.V, S.K. Maharana, Abdul Sharief et al. (2018), Study of effects of rotational rate of a cylinder on the film boiling phenomenon of water undergoing phase change over two heated cylinders in tandem arrangement, International Journal of Modern Engineering Research, Vol 8 (pp 62-70)

[6]. Mahir N and Altac Z et al.(2008), Numerical investigation of convective heat transfer in unsteady flow past two cylinders in tandem arrangements, Int J Heat Fluid Flow, Vol 29 (pp 1309-1318).

[7]. Nicol AA \& McLean JT et al.(1968), Boiling Heat Transfer from a Rotating Horizontal Cylinder, Canadian J. Chem. Eng, Vol. 46, (pp 304-308).

[8]. Anderson IT \& Saunders OA et al.(1953), Convection from an Isolated Heated Horizontal Cylinder Rotating About Its Axis, Proc. Roy. Soc, A, (pp 217- 555).

[9]. Becker KM et al.(1963), Measurements of Convective Heat Transfer from a Horizontal Cylinder Rotating in a Tank of Water, Int. J. Heat Mass Transfer, Vol. 6, (pp 1053-1062).

[10]. Etemad GA et al.(1955), Free Convection Heat Transfer from a Rotating Cylinder to Ambient Air, With Interferometric Study of Flow, Trans. ASME, Vol. 77, ( pp 1283).

[11]. Dropkin D \& Carmi A et al.(1956), Natural Convection Heat transfer from a Horizontal Cylinder Rotating in Air, Heat Transfer and Fluid Mechanics Institute, Stanford University. [12]. Lienhard J H et al.(1963), A Semi-Rational Nucleate Boiling Heat Flux Correlation, Int. J Heat Mass Transfer, Vol. 6 (pp 215-219).

[13]. Shih-I Tang et al.(1970), A study of nucleate boiling heat transfer over a horizontal rotating cylinder, University of Windsor, Electronic theses and dissertations.

[14]. Ding H, Shu C and Yeo KS et al.(2007), Numerical simulation of flows around two circular cylinders by meshfree least square-based finite difference methods, Int J Numer Methods Fluids (pp 305-332).

[15]. V.K. Dhir et al.(1991), Nucleate and Transition Boiling Heat Transfer under Pool and External Flow Conditions, International Journal of Heat Fluid Flow, vol. 12 (pp 290314).

[16]. I.L. Pioro, W. Rohsenow and S.S. Doerffer et al.(2004), Nucleate Pool-boiling Heat Transfer. I: Review of Parametric Effects of Boiling Surface, International Journal of Heat Mass Transfer, Vol. 47 (pp 5033-5044). 